Muro de la Investigación, 2020(2): julio-diciembre

ISSN:2523-2886

Doi: https://doi.org/10.17162/rmi.v5i2.1324

\title{
La procrastinación académica: teorías, elementos y modelos
}

\section{Academic procrastination: theories, elements and models}

\author{
Anthony Santiago Ayala Ramírez ${ }^{1 \mathrm{a}}$, Ruth Yoselin Rodríguez Diaz ${ }^{2 \mathrm{a}}$, \\ Wilma Villanueva Quispe ${ }^{3 a}$, Melva Hernández Garcia ${ }^{4 a}$ \\ Madeleine Campos Ramirez ${ }^{5 a}$ \\ ORCID iD: https://orcid.org/0000-0001-8138-8235 3a \\ ORCID iD: https://orcid.org/0000-0001-6685-45414a \\ ORCID iD: https://orcid.org/0000-0002-4046-2176
}

Universidad Peruana Unión, Perú ${ }^{12345}$

Recibido: 03 de diciembre de 2019

Aceptado: 02 de abril de 2020

\begin{abstract}
Resumen
El objetivo de esta investigación es analizar las teorías, elementos y modelos de la procrastinación en el contexto de los estilos negativos de aprendizaje, del bajo rendimiento académico, la falta de resolución de conflictos, la indebida toma de decisiones, y la postergación de la realización de las actividades; factores que debilitan de diversas maneras al proceso educativo. En este sentido, el análisis de las teorías, los elementos y los modelos de la procrastinación permiten conocer y abordar los problemas que ella genera en los estudiantes, cuyas consecuencias son multidimensionales.
\end{abstract}

Palabras clave: Procrastinación, aprendizaje, educación.

\begin{abstract}
The objective of this research is to analyze the theories, elements and models of procrastination in the context of negative learning styles, low academic performance, lack of conflict resolution, improper decision-making, and postponement of implementation. of the activities; factors that weaken the educational process in various ways. In this sense, the analysis of the theories, elements and models of procrastination make it possible to understand and address the problems that it generates in students, the consequences of which are multidimensional.
\end{abstract}

Key words: Procrastination, learning, education.

\section{Introducción}

\footnotetext{
${ }^{1}$ Correspondencia a los autores: anthonyayala@upeu.edu.pe,ruthrodriguez@upeu.edu.pe,
} wilvil@upeu.edu.pe,melvahernadez@upeu.edu.pe 
El mundo es cambiante y competitivo, le exige al ser humano logros y desafíos laborales, sociales y académicos; en este sentido, una meta muy importante y relevante es la formación mediante el proceso de aprendizaje, involucrando hábitos, valores y virtudes (Figuera y Torrado, 2015), los cuales son influyentes sobre la formación y rendimiento académico del niño; en este, intervienen componentes positivos y negativos en la organización de tareas. Mientras la pereza (como hábito), para muchos, puede ser normal, para algunos profesionales significa la presencia de un serio problema que afecta el proceso educativo, causando un bajo rendimiento y procrastinación. Una consecuencia fundamental de este proceso es el rendimie nto académico, el cual se ha visto afectado por diferentes motivos, generando consecuencias negativas durante el proceso educativo.

Los factores esenciales del bajo rendimiento académico son: aspectos negativos, deficiencias de los casos del proceso educativo, dilación o procrastinación académica, considerada esta última como una tendencia muy común durante los últimos años (Badia, Dezcallar, y Cladellas, 2018). En concepto, la procrastinación es la inclinación a posponer o retrasar la finalización de una labor, dejando al margen responsabilidades, decisiones y entrega de tareas desarrolladas (Piers Steel, 2007). Este tipo de conducta es un simple error, el cual no debe tomarse muy livianamente, pues al procrastinar cualquier tarea se pierde el foco central de la meta de aprendizaje.

En el ámbito latinoamericano, según las investigaciones consultadas, más de un 90\% de estudiantes procrastina, aunque no siempre con carácter crónico (Natividad, 2014); asimismo, Guzmán (2013) detalla que entre el $80 \%$ y el $95 \%$ de los estudiantes procrastina; de estos, el 70\% se considera un procrastinador y casi el 50\% procrastina de manera consistente y problemática. Se muestra que del $20 \%$ al 25\% de la población general en América son procrastinadores crónicos (Díaz, 2019).

Por otro lado, Ferrari, Johnson y McCown (1995) afirman que los diversos motivos por los cuales los estudiantes procrastinan son: dificultad del cumplimiento de horarios, desempeños y competencias del estudiante, cuyos motivos influyen sobre el rendimiento académico y es afectado el proceso educativo.

Por su parte, la Organización para la Cooperación y el Desarrollo Económicos (OCDE) publicó un informe sobre la base de los resultados de la prueba PISA 2012; el informe detalla que el Perú tiene el más alto porcentaje de estudiantes de 15 años, quienes no alcanzaron el nivel básico establecido por la OCDE en lectura (60\%), en ciencia (68,5 $\%$ ), y en matemáticas (74,6 \%). La UNICEF, el año 2015, determinó que el 33\% de los niños tiene comprensión lectora y solamente el $16.8 \%$ comprende matemática. 
A la luz de estos antecedentes, el presente artículo realiza un análisis sobre la procrastinación, sus teorías, elementos y modelos, con el propósito de observar la procrastinación relacionada con los aspectos académicos, y como repercute sobre el desarrollo y el aprendizaje del estudiante.

\section{La procrastinación, conceptos}

La procrastinación académica consiste en posponer el desempeño en las tareas académicas. Es un fenómeno multifacético, pues parece involucrar aspectos afectivos, cognitivos y conductuales (Vargas, 2017). Chan (2011) añade que la procrastinación es una afectación de la toma de decisiones y de la resolución de conflictos, durante el momento de decidir si culmina los deberes académicos o satisfacer los requerimientos en su ambiente.

Por su parte, Steel (2007) ha definido la procrastinación como un retraso voluntario de un curso de acción previsto, aunque el retraso sea peor. Rothblum, Solomon, y Murakami (1986) creen que la procrastinación académica es un retraso irracional o aplazamiento de las tareas académicas, hasta experimentar una ansiedad considerable. Es por ello que los conceptos corrientes de procrastinación académica la definen como una especie de falta de motivación, muy relacionada con el fracaso de la autorregulación; en consecuencia, se perciben los bajos niveles de autoeficacia y autoestima académica y un alto nivel de estrés y enfermedad.

Sobre la procrastinación académica en estudiantes más pequeños antes de los 12 años, las referencias son escasas. Explorar con éxito la procrastinación académica en edades más tempranas presenta mucha dificultad. Los estudios analizados son de educación secundaria, no aparecen antes de esta etapa. Aunque, Dezcallar, Clariana, Cladellas, Badia, y Gotzens (2014) muestran que los alumnos varones de educación primaria de 6 a 12 años, quienes practican actividades extraescolares de deporte y las alumnas de la misma etapa educativa quienes realizan actividades extraescolares de artes y música, procrastinan significativamente menos que sus pares que no realizan este tipo de actividades fuera de la escuela.

\section{Teorías de la procrastinación}

\section{Teoría de la motivación temporal (TMT)}

Esta teoría, propuesta por Steel y König (2006), propone la unión de la teoría motivacional con las teorías económicas. 


Motivación $=\frac{\text { Expectativa } \times \text { Valor }}{1+\text { Impulsividad } \times \text { Retraso }}$

Los factores referidos por la teoría de motivación, según la ecuación presentada, son: motivación/utilidad (motivación baja), expectativa (más al fracaso), valor (valor alto y procrastinación mínima), impulsividad (falta de impulso) y retraso (pérdida de tiempo). Para no procrastinar se tiene en cuenta una "motivación" alta, seguida de una "expectativa" de éxito con un "valor" suficiente de la tarea; así como no dejar la "impulsividad" para dejar de lado y organizar el "retraso" de la tarea; si se cumple esta ecuación de forma positiva, la procrastinación no sería posible; caso contrario, el único resultado sería la procrastinación y una catástrofe para el aprendizaje y el proceso educativo.

\section{Teoría de la psicodinámica}

La perspectiva psicodinámica cree que la procrastinación es el incumplimiento de trabajos primordiales, generadores de gran angustia y son tomados como alertas amenazantes. Inconscientemente, se comenzarían a emplear múltiples mecanismos de defensa para disminuir el malestar y evitar dicha tarea (Ferrari, Barnes y Steel, 2009).

Burka y Yuen (2008) detallan que la procrastinación ocurre cuando aparece el temor del niño, al pensar que sus actos tendrán consecuencias desfavorables; el pequeño evitará ser castigado; el aumento de la cantidad de tareas escolares, las fechas límite, las responsabilidades en casa, determinan una diferencia en los niveles de ansiedad de los niños, terminarían postergando sus quehaceres.

Angarita (2012) afirma que la procrastinación es el resultado de un estilo de crianza autoritario. En la adolescencia, se da la idea de que el tiempo pasa muy rápido, con todos los cambios físicos; estos generan una sensación de que nada puede volver. Estas sensaciones, más la tendencia a imaginar el futuro, y el miedo a madurar, llevan al adolescente a procrastinar. En un joven, a los 20 años promedio, esta perspectiva se reemplaza por la idea de que el tiempo es insuficiente para lograr las metas propuestas, las oportunidades son únicas y estarían perdiéndose, generando altos niveles de ansiedad; esto produce la conducta de procrastinar, y se puede convertir en una conducta repetitiva hasta la vejez si no se controla a tiempo.

\section{Teoría del conductismo}


Según Álvarez (2010), esta teoría describe que las conductas que se mantienen o se repiten son forzadas; es decir, permanecen por sus consecuencias. Las personas quienes procrastinan y se mantienen realizando esta conducta de posponer actividades, no han tenido éxito muchas veces en sus objetivos, por los factores que les han permitido continuar procrastinando sin consecuencias desfavorables (Burka y Yuen, 2008).

\section{Teoría racional emotiva conductual (TREC)}

Para Ellis y Knaus (en Álvarez, 2010), este modelo propone que los desórdenes emocionales se originan por las creencias irracionales sobre las situaciones en las que vive, cuyas consecuencias son emocionales o conductuales desfavorables. La procrastinación comienza con las creencias irracionales sobre la tarea concluida satisfactoriamente, debido a deficiente tolerancia a la frustración. Se plantean metas altas y poco reales, cuyos resultados son opuestos a los esperados. En consecuencia, se desencadenan emociones incómodas, comenzará a demorarse la realización de dicha tarea, hasta la complicación e imposibilidad de realizarlas; se eludirá el auto cuestionamiento sobre sus habilidades y capacidades.

García (2009) muestra un ejemplo de hipótesis básica de ese modelo, el A-B-C de Albert Ellis, el creador de la terapia racional emotivo-conductual, quien ilustra una situación de procrastinación. Se especifica: "A" se refiere al evento Activador, "B" contiene las creencias al respecto, $\mathrm{y}$ " $\mathrm{C}$ " refleja las consecuencias emocionales $\mathrm{y}$ conductuales.

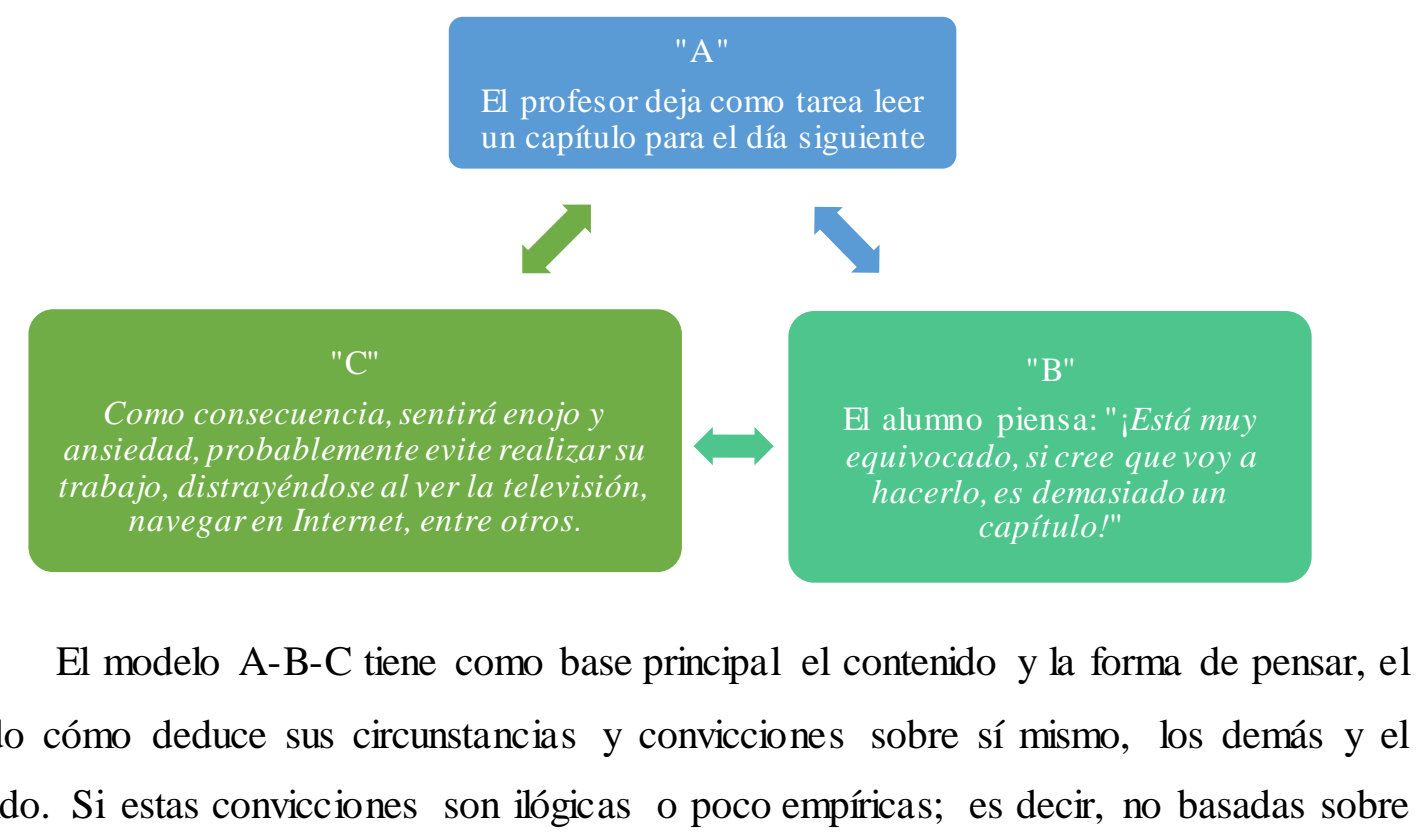


datos objetivos y verificables, dificultan el logro de los objetivos, y esto recibe el nombre de "irracional", porque se hace mal razonamiento. Según dicho modelo, los estudiantes están involucrados en la procrastinación, por una forma errónea de razonar al recibir un componente activador, el cual destruye sus expectativas para realizar una tarea propuesta.

\section{Elementos de la procrastinación}

A partir de su investigación, Steel (2007) señala cuatro factores motivacionales en la postergación de actividades:

a) Expectativa: este factor refiere la forma de realizar una tarea.

b) Valoración: valor o precio dado a algunas tareas más agradables que otras, cuya base es una evaluación de factores: tiempo y herramientas, el objetivo de mayor valor es la obtención de un "premio" en menos tiempo y sin tanto esfuerzo.

c) Impulsividad: muestra al individuo poco atraído por la tarea, falta de motivación.

d) Demora de la satisfacción: elemento o factor relacionado con el tiempo de respuesta y algunos beneficios de la repetición de esta conducta; al procrastinador le resulta gratificante un resultado inmediato que un resultado con demora.

Por otro lado, Burka y Yuen (2008) abordan un elemento importante relacionado con la procrastinación: el temor al éxito. Detallan cogniciones que explican la evasión del éxito: a) "El éxito demanda demasiado esfuerzo, debo retirarme": "Tómalo o déjalo", "Fobia al compromiso", "Me volveré un adicto al trabajo"; b) "El éxito es peligroso: alguien siempre sale herido": "Podría herir a alguien", "Me pueden herir"; "El éxito está fuera de los límites": "No merezco el éxito", "El éxito no es mi destino".

Ferrari y Díaz (2007) argumentan que existen dos factores importantes estrechamente relacionados con la procrastinación: el autoconcepto (o autocontrol) y la autopresentación; el primero es el control total de los pensamientos y sentimientos sobre sí mismo, no tiene relación con los procrastinadores, son confiables en tareas logradas a tiempo, las tendencias de dilación están relacionadas con la baja dependencia, la baja integridad y baja fiabilidad en completar una tarea. La auto presentación describe que los procrastinadores están preocupados por su imagen pública y su autoconciencia, quienes tienden a manipular su impresión pública, por medio de la excusa y la justificación. 
En otro orden, Clariana (2013) describe el cheating o el engaño, considerado un factor que promueva la procrastinación académica, los estudiantes son más impulsivos y sus notas son bajas; esto puede deberse a diversas causas: falta de planificación del profesor, personalidad del estudiante, falta de motivación, variedad de estudiantes. El estudiante que practica el cheating tiene dos situaciones: el estudiante engaña a los demás y se engaña a sí mismo, experimentando procrastinación de por vida, llena de engaños y falsas expectativas.

\section{Modelos y características de la procrastinación.}

Algunos individuos que procrastinan presentan rasgos de personalidad muy marcados: impulsividad, perfeccionismo, dependencia de los demás, y cometer un error puede ser un fracaso total (Quant y Sánchez, 2012). Así, los individuos postergan de vez en cuando alguna actividad por dejadez o poco compromiso; los procrastinadores dan por hecho no actuar; hacen de esta conducta un hábito, aparecen las excusas, convertidas en un estilo de vida mal adaptado. Además, existe la relación entre la procrastinación crónica y los rasgos de personalidad pasivos agresivos, trastornos: déficit de atención e hiperactividad, trastorno obsesivo compulsivo y otros (Ferrari, Johnson, y McCown, 1995).

Por otro lado, existe diferencia entre los estudiantes que procrastinan y quienes no lo hacen; los segundos logran tener una identidad definida, se preocupan en cómo se sienten ellos mismos y no en cómo otras personas pueden opinar o percibir de ellos; existe mejor compromiso hacia sí mismos.

Entre los modelos de procrastinación, algunos investigadores lo plantean como tipos de procrastinadores o modelos de procrastinación, considerados similares, porque la persona que procrastina es considerada procrastinador del tipo al que pertenece. Para Steel (2007) existen cuatro tipos de procrastinadores, resumidos a continuación:

$\begin{array}{llll}\begin{array}{l}\text { Thrill Seekers } \\ \text { (Busca mociones }\end{array} & \begin{array}{l}\text { Impulsives } \\ \text { (Impulsivos) }\end{array} & \begin{array}{l}\text { Undecided } \\ \text { (Indecisos) }\end{array} & \begin{array}{l}\text { Avoiders } \\ \text { (Evitadores) }\end{array} \\ \begin{array}{l}\text { Disfruta terminar } \\ \text { tarea justo a tiempo }\end{array} & \begin{array}{l}\text { Sin disciplina y } \\ \text { fácil distracción }\end{array} & \begin{array}{l}\text { Dificultad en tomar } \\ \text { decisiones, se } \\ \text { estancan. }\end{array} & \begin{array}{l}\text { Temor a fallar o } \\ \text { desaprobación de } \\ \text { los demás }\end{array}\end{array}$

El autor describe que estos tipos de procrastinadores tienen características diferentes y el fin de estos siempre es la dilación de forma esporádica o crónica, también 
los procrastinadores impulsivos tienen la procrastinación como un hábito adquirido por la conducta repetitiva de diferentes tareas. Por otro lado, Chun-Chu y Choi (2005) dividen la procrastinación solamente en dos modelos, los cuales son denominados: Procrastinación Pasiva y Procrastinación Activa, se los resume de la siguiente manera:

Procrastinación Pasiva

- Son más comunes

- Son indecisos

- No terminan lo asignado a tiempo

- Sin intención de posponer actividades

- Incapaces de tomar decisiones rápidamente

\section{Procrastinación Activa}

- Trabajan bajo presión

- Deciden con libertad el aplazar tareas

- No existe estrés con actividades a largo plazo

- El tiempo es su mejor aliado

Los procrastinadores activos son igual de propensos en postergar actividades al igual que los pasivos; los procrastinadores activos son más similares a los individuos que no procrastinan por algunas características: uso intencional del tiempo, control del tiempo, creencias de autoeficacia, estilos de afrontamiento y resultados, incluyendo el rendimiento académico; ambos tipos de procrastinadores influyen significativamente en el bajo rendimiento y el aprendizaje del estudiante. Casi todos los seres humanos tendemos a ser procrastinadores pasivos o tradicionales, por lo menos una vez en la vida, dando a la procrastinación un papel normal en lo cotidiano del ser humano. Ferrari et al. (1995) dividen en tres grupos la procrastinación: 1. procrastinación aurosal, 2. procrastinación evitativa y 3. procrastinación decisional. Se detalla a cada uno de estos modelos de la siguiente manera:
Aurosal
Evitativo
Decisional

- Alto nivel de búsqueda de sensaciones

- Satisfacción al hacer la tarea en corto plazo

- Corre el riesgo al fracaso
- Evita no enfrentarse a una tarea

- Posterga por temor a fallar

- Miedo a enfrentarse con limitaciones
- Simplemente procrastina en la toma de una decisión para la ejecución de una tarea

La similitud de los tres tipos de procrastinadores es la dilación o aplazamiento de tareas; sus diferencias responden a causas de la procrastinación académica, dichas causas son psicológicas, afectan el desarrollo educativo o proceso educativo.

\section{Dimensiones de procrastinación académica Autorregulación académica}


Pozo, Monereo y Castelló (2001) detallan la autorregulación como una conducta estratégica de aprendizaje, considerada un mecanismo intrapsicológico, reflejado en conductas y actividades de pensamiento destinados a gestionar los propios recursos personales, a fin de conseguir un objetivo de aprendizaje.

Baumeister, Vohs, DeWall y Zhang (2007) declararon que la autorregulación refería la capacidad para cambiar su propio comportamiento relacionado con el aprendizaje. La autorregulación académica no es una habilidad mental sino un proceso autodirigido, durante el cual los estudiantes cambian sus habilidades mentales en una habilidad académica.

Por su parte, Pintrich (2000) define la autorregulación como un proceso activo y constructivo, mediante el cual el alumno establece objetivos, supervisa su aprendizaje y controla su motivación, comportamiento y cognición. Por ello, la autorregulación académica es una acción reguladora que el estudiante cumple durante varios momentos de su proceso de aprendizaje. Para esta acción reguladora es necesario, primero, el conocimiento de lo que se hace y se conoce; y segundo, el conocimiento al que se accede por medio de la metacognición. En otras palabras, un estudiante difícilmente se autorregula académicamente, si no posee conocimiento sobre sí mismo, sus emociones y sus estrategias de aprendizaje.

Postergación de actividades

Dominguez, Villegas, y Centeno (2014) crean el concepto de postergación: la tendencia de aplazar a menudo o siempre una actividad, en muchos de los casos se produce ansiedad, baja autoeficacia y estrés, por la entrega de trabajos, y, en consecuencia, traerá una nota o calificación negativa.

Por otro lado, la postergación de actividades es el sinónimo de procrastinación, la cual es una decisión intencionada, pasa de fugaz, lleva a postergar sin necesidad, dejar incompleta alguna tarea; dicha tarea la sustituye por otra actividad de prioridad mínima, sabiendo las consecuencias negativas y posibles desventajas (Palacios y Palos, 2007). Por eso, Rothblum et al. (1986) declaran que existe una alta predisposición a postergar algunas tareas académicas, por lo cual esto afecta a los individuos que se desempeñan en el ámbito académico, ya que estos presentan sus tareas a un tiempo no estimado, planteado o requerido por el maestro o tutor.

\section{Conclusiones}


Se concluye que la procrastinación es el acto de postergar actividades de manera irresponsable, sabiendo las consecuencias que ello acarrea y los resultados negativos en el proceso educativo de enseñanza aprendizaje. El análisis de las cuatro teorías describe la procrastinación como un mal hábito. Una es la teoría motivacional (Steel y König, 2013), la cual describe mediante una ecuación los elementos que influyen sobre la motivación: a más motivación menos procrastinación y viceversa. La teoría de la psicodinámica afirma que la procrastinación es el resultado de situaciones experimentadas durante la niñez; la angustia, el estilo de crianza, la ansiedad y la acumulación de tareas, conllevan la postergación de tareas; el individuo hecho adulto repite estas actitudes y el tiempo es un factor importante en situaciones no necesarias. Para la teoría conductual de Skinner, la procrastinación es una conducta repetitiva; esta conducta no deja consecuencias demasiado desfavorables, se repite y forma procrastinadores. La teoría racional emotiva conductual de Ellis está involucrada en aspectos psicológicos (forma de crianza), cognitivos (uso adecuado de la razón) y conductuales (acción de cómo se desarrolla una circunstancia), esta última postura está completa y abarca los aspectos, cuya procrastinación es influida.

Se observó que los factores de la procrastinación son: expectativa, valoración, impulsividad, demora de la satisfacción, temor al éxito, autocontrol, autopresentación, y, por último, el cheating o engaño. Estos determinan las dimensiones de la procrastinación: autorregulación y postergación de actividades, consideradas opuestas, una persona que se autorregula no posterga alguna actividad, sino más bien tiende a cumplirla.

Los modelos de procrastinación tienen una característica principal: todos los modelos o tipos de procrastinación completan su proceso. Steel presenta cuatro tipos de procrastinador: indeciso, impulsivo, buscador de emociones y evitador. Para Chun-Chu y Choi (2005), los procrastinadores son pasivos y activos. Ferrari presenta tres tipos de procrastinación: procrastinación aurosal, evitativa y decisional. En suma, es más relevante el pensamiento de Chun-Chu y Choi (2005), quienes dividen la procrastinación de manera dicotómica, detallando la procrastinación solo en dos modelos.

\section{Referencias}

Álvarez, Ó. (2010). Procrastinación general y académica en una muestra de estudiantes de secundaria de Lima metropolitana. Revista Persona, 9(13), 159-177.

Angarita, L. (2012). Aproximación a un concepto actualizado de la procrastinación. 
Revista iberoamericana de psicología: ciencia y tecnología, 5(2), 85-94. Retrieved from https://dialnet.unirioja.es/descarga/articulo/4905094.pdf\%5Cn

Badia, M. del M., Dezcallar, T., \& Cladellas, R. (2018). Uso lúdico de las TIC, procrastinación y género: un estudio con alumnos de educación primaria. Aloma: Revista de psicologia, ciències de l'educació i de l'esport, 36(2), 33-40.

Baumeister, R. F., Vohs, K. D., DeWall, C. N., y Zhang, L. (2007). How emotion shapes behavior: Feedback, Anticipation, and Reflection, Rather than Direct Causation. personality and social psychology review, 11(2), 167-203. https://doi.org/10.1177/1088868307301033

Burka, J., y Yuen, L. (2008). Procrastination why you do it, what to do about it now. Massachusetts, Estados Unidos de America: Da Capo Press.

Carranza, R., y Ramírez, A. (2013). Procrastinación y características demográficas asociados en estudiantes universitarios. Apuntes Universitarios, 3(2), 95-108. https://doi.org/10.17162/au.v0i2.43

Chan, L. A. (2011). Academic procrastination as predictor of academic performance among young people with higher education. Unife, 7(1), 53-62.

Chun Chu, A. H., \& Choi, J. N. (2005). Rethinking procrastination: Positive effects of "active" procrastination behavior on attitudes and performance. Journal of Social Psychology, 145(3), 245-264. https//doi.org/10.3200/SOCP.145.3.245-264

Clariana, M. (2013). Personalidad, procrastinación y conducta deshonesta en alumnado de distintos grados universitarios. Electronic journal of research in educational psychology, 11(2), 451-472. https//doi.org/10.14204/ejrep.30.13030

Dezcallar, T., Clariana, M., Cladellas, R., Badia, M., y Gotzens, C. (2014). La lectura por placer: su incidencia en el rendimiento académico, las horas de televisión y las horas de videojuegos. OCNOS: Revista de estudios sobre lectura, 12, 107-116. Retrieved from http://www.redalyc.org/articulo.oa?id=259132660005

Diáz, J. (2019). Procrastination: A review of scales and correlates. Revista iberoamericana de diagnostico y evaluacion psicologica, 51(2), 43-60. https://doi.org/10.21865/RIDEP51.2.04

Dominguez, S., Villegas, G., y Centeno, S. (2014). Procrastinación académica: validación de una escala en una muestra de estudiantes de una universidad privada. Liberabit. Revista de psicología, 20(2), 293-304.

Ferrari, J., \& Díaz, J. (2007). Perceptions of self-concept and self-presentation by procrastinators: further evidence. The spanish journal of psychology, 10(1), 91-96. 
Ferrari, J., Johnson, J., y McCown, W. (1995). Procrastination and task avoidance: theory, research, and treatment. Nueva York, Estados Unidos de America: (Springer Science+Business Media. https://doi.org/10.1007/978-1-4899-0227-6

Ferrari, J. R. (2000). Procrastination and attention: factor analysis of attention deficit, boredomness, intelligence, self-esteem, and task delay frequencies. Journal of social behavior and personality, 15(5), 185-196.

Ferrari, J. R., Barnes, K. L., y Steel, P. (2009). Life regrets by avoidant and arousal procrastinators: why put off today what you will regret tomorrow? Journal of individual differences, 30(3), 163-168. https://doi.org/10.1027/1614-0001.30.3.163

Figuera, P. y Torrado, M. (2015). La adaptación y la persistencia académica en la transición en el primer año de universidad: el caso de la Universidad de Barcelona. Opción, 31(6).

García, C. (2009). Comprendiendo la procrastinación con el modelo ABC de Albert Ellis. Gaceta de la escuela de medicina Justo Sierra, 2(1), 2-5.

Guzmán, D. (2013). Procrastinación: una mirada clínica. 0-51. Recuperado de: $\mathrm{http} / / \mathrm{www}$. isep.es/tesina/procrastinacion/

Milgram, N. (1992). El retraso: una enfermedad de los tiempos modernos. Boletín de psicología, (35), 83-102.

Natividad, L. (2014). Análisis de la procrastinación en estudiantes. (Tesis doctoral). Univestat de Valéncia, Valencia, España.

Navarro, R. E. (2003). El rendimiento académico: concepto, invetigación y desarrollo. REICE - Revista electrónica iberoamericana sobre calidad, eficacia y cambio en educación, 1(2), 1-15. https://doi.org/2152

Palacios, J., \& Palos, P. (2007). Desempeño académico y conductas de riesgo en adolescentes. Revista de educación y desarrollo, 7, 5-16.

Pintrich, P. (2000). The Role of goal orientation in self-regulated learning. Handbook of self-regulation, 451-502. Recuperado de: http://cachescan.bcub.ro/ebook/E1/580704/451-529.pdf

Pozo, J. I., Monereo, C., y Castelló, M. (2001). El uso del conocimiento. El aprendizaje Estratégico, 1-27.

Quant, D., \& Sánchez, A. (2012). Procrastinación , Procrastinación Académica : Concepto e Implicaciones. Revista Vanguardia Psicológica, 3(1), 45-59. Recuperado de: http//www.academia.edu/10370305/PROCRASTIN ACIÓN_PROCRASTINACIÓ 


\section{N_ACADÉMICA_CONCEPTO_E_IMPLICACIONES}

Rothblum, E. D., Solomon, L. J., y Murakami, J. (1986). Affective, cognitive, and behavioral differences between high and low procrastinators. Journal of counseling psychology, 33(4), 387-394. https//doi.org/10.1037/0022-0167.33.4.387

Steel, P, y König, J. (2013). Theories of motivation integrating. Academy ofmanagement, 31(4), 889-913.

Steel, Piers. (2007). The nature of procrastination: A meta-analytic and theoretical review of quintessential self-regulatory failure. Psychological Bulletin, 133(1), 65-94. https://doi.org/10.1037/0033-2909.133.1.65

Steel, Piers, y König, C. J. (2006). Integrating theories of motivation. Academy of management review, 31(4), 889-913. https//doi.org/10.5465/AMR.2006.22527462

Vargas, M. A. P. (2017). Academic procrastination: the case of mexican researchers in psychology. American journal of education and learning, 2(2), 103-120. https://doi.org/10.20448/804.2.2.103.120 